Published in final edited form as:

J Neurol Neurosurg Psychiatry. 2009 October ; 80(10): 1143-1145. doi:10.1136/jnnp.2008.155846.

\title{
Emotion Recognition in Progressive Supranuclear Palsy
}

\author{
B C P Ghosh ${ }^{1,2}$, J B Rowe ${ }^{1,2}$, A J Calder ${ }^{2}$, J R Hodges ${ }^{2,3}$, and T H Bak ${ }^{4}$ \\ ${ }^{1}$ Department of Clinical Neurosciences, Cambridge University. CB2 2QQ UK \\ 2 Medical Research Council Cognition and Brain Sciences Unit, Cambridge. CB2 7EF UK \\ ${ }^{3}$ Prince of Wales Medical Research Institute, Sydney, New South Wales 2031, Australia \\ ${ }^{4}$ University of Edinburgh, 7 George Square, Edinburgh. EH8 9JZ UK
}

\begin{abstract}
Progressive supranuclear palsy (PSP) is an atypical parkinsonian syndrome characterised by akinesis, rigidity, falls, supranuclear gaze palsy, and cognitive, particularly executive dysfunction. This study examined the extent to which emotion recognition is affected by PSP. Although deficits in the recognition of emotion have been reported in several diseases which share clinicopathological characteristics with PSP, it has never been studied systematically in PSP.

We studied 24 patients with probable or definite PSP and matched healthy controls, on tests of facial identity and facial emotion recognition. Patients were not impaired in recognising famous faces, but they showed significant deficits in the recognition of emotions, particularly negative emotions. Moreover, emotion recognition was strongly correlated with the severity of other cognitive deficits in PSP, but not disease duration.
\end{abstract}

We suggest that deficits in emotion recognition form an integral part of the cognitive spectrum of the disease. The findings point to the pathological involvement of key regions necessary for the processing of emotions and to a subtype of PSP with cognitive and emotion recognition impairments. The acknowledgement of deficits in emotion recognition is important for management of both patients and their carers.

\section{Keywords}

progressive supranuclear palsy; emotion recognition; Dementia Rating Scale; Addenbrookes Cognitive Examination

\section{INTRODUCTION}

Progressive supranuclear palsy (PSP) is a severe, progressive, neurodegenerative disease, characterised by a symmetrical, akinetic-rigid syndrome, supranuclear paresis of vertical gaze, executive dysfunction and apathy [1-4]. There is evidence of disordered social interactions in many patients with PSP, including difficulties with relationships in the family and problems with communication [5]. Disordered social interactions may arise from diverse reasons, such as deteriorating physical function or verbal communication [6].

\footnotetext{
Correspondence should be addressed to: Dr B Ghosh bcpg1@cam.ac.uk Box 83, Department of Neurosciences, Addenbrookes Hospital, Cambridge. CB2 2QQ Telephone 01223216509 Fax 01223217909.

Competing interests:

None
} 
However, it is also possible that the pathology in PSP directly affects the neurocognitive processes of emotion.

Impaired recognition of emotion occurs in other diseases that share clinical and pathological features with PSP, such as frontotemporal dementia (FTD), Parkinson's disease (PD) and Huntington's disease (HD). Patients with HD have particular problems in disgust recognition [7], whereas PD [8, 9] and FTD [10] are generally associated with more global impairments. Our aim was to investigate whether PSP was associated with similar, but previously unidentified, deficits in emotion recognition.

\section{METHODS}

24 patients seen at the Cambridge University Disorders of Movement and Cognition clinic (DMC) between 1996 and 2004 were included. They were diagnosed with probable PSP [11], by an experienced neurologist (THB or JRH). Exclusion criteria included: age greater than 75; other coexisting neurodegenerative conditions and inability to participate in neuropsychological assessment. All patients and controls provided informed consent. Seven patients had donated their brains for analysis and were confirmed by post mortem neuropathology to have PSP [11, 12]

Matched control subjects were recruited from the volunteer panel of the Medical Research Council Cognition and Brain Sciences Unit, Cambridge: 23 controls for the famous faces test and 23 different controls for the emotion recognition test. In addition to demographic data, cognitive screening was carried out in patients, using the Dementia Rating Scale (DRS) [13] and the Addenbrookes Cognitive Examination (ACE) [1, 14].

In the famous faces test, subjects viewed the faces of 30 famous celebrities, intermixed with 10 unfamiliar faces [15]. Marks were given for correctly identifying famous people and for correctly rejecting non-famous people.

The Ekman 60 emotion recognition test [15] used stimuli from the Ekman and Friesen pictures of facial affect [16]. Sixty pictures depicting the six primary emotions (anger; fear; disgust; happiness; sadness and surprise) were presented in random order. Subjects were asked to pair the emotion displayed in each photograph with one of six emotion labels displayed on the screen.

Statistical analysis used SPSS v 12 (SPSS Inc., Chicago, IL). Demographic differences between patients and controls were compared with one-way ANOVA and between the two control groups with $t$ tests. The duration of the disease from onset to testing, as well as the stage of disease were calculated. The stage of the disease was defined as the point in the disease course, between onset and death, at which the testing was carried out (available for 19/24 patients). Eighteen patients completed the famous faces test. Demographics, emotion recognition and cognitive scores for these patients were compared using an independent samples t-test with the 6 patients who had not completed this test. For the famous faces and the emotion tests, we used repeated measures analysis of variance (rm-ANOVA) with post hoc $\mathrm{t}$ tests and Greenhouse-Geisser correction for non-sphericity where appropriate. Correlation analyses were used to investigate co-factors affecting patients' scores on the emotion recognition test.

\section{RESULTS}

Demographic details for our patients and controls are given in table 1. There was no significant difference between our patients and controls in terms of age $(\mathrm{F}<1)$, education $(\mathrm{F}<1)$ or gender $(\mathrm{F}=1.74, \mathrm{df}=2,67, \mathrm{p}=\mathrm{ns})$, or between our two control groups in terms of age 
$(\mathrm{t}<1)$, gender $(\mathrm{t}<1)$ or education $(\mathrm{t}=-1.12, \mathrm{df}=44, \mathrm{p}=\mathrm{ns})$. All patients completed the Ekman 60 test. Eighteen completed the famous faces test. There was no significant difference between those who did $(\mathrm{n}=18)$ and $\operatorname{did}$ not $(\mathrm{n}=6)$ complete the test (age $(\mathrm{t}<1)$, education $(\mathrm{t}<1)$, gender ( $\mathrm{t}=-1.35, \mathrm{df}=11$ (adjusted due to unequal variances), $\mathrm{p}=\mathrm{ns}$ ), emotion recognition score $(t<1)$, ACE score $(t<1)$, DRS score $(t<1)$, duration $(t<1)$, stage of disease $(\mathrm{t}=-1.21, \mathrm{df}=17, \mathrm{p}=\mathrm{ns}))$.

Rm-ANOVA showed a main effect for test $(\mathrm{F}=14.0, \mathrm{df}=1,39, \mathrm{p}<0.001)$ but no main effect for group $(\mathrm{F}=<1)$ or interaction between test and group $(\mathrm{F}=3.6, \mathrm{df}=1,39, \mathrm{p}=\mathrm{ns})$. When analysed post hoc, there was no significant difference between participants' ability to recognise famous faces $(\mathrm{t}=1.5, \mathrm{df}=39, \mathrm{p}=\mathrm{ns})$ or correctly reject unfamiliar faces $(\mathrm{t}=-1.3$, $\mathrm{df}=39, \mathrm{p}=\mathrm{ns}$ ). These results were unchanged when the same data were arcsin-transformed to correct for near ceiling effects. The lack of significant difference between groups for both familiar and unfamiliar faces indicates that the patients' ability to recognise familiar faces did not reflect a bias to respond 'familiar'.

For the emotion recognition test, there was a main effect of emotion $(\mathrm{F}=38.7, \mathrm{df}=4.2,187.4$, $\mathrm{p}<0.001)$ and an emotion $\mathrm{x}$ group interaction $(\mathrm{F}=2.7, \mathrm{df}=4.2,187.4, \mathrm{p}=0.029)$ in the within subjects analysis. There was a significant effect for group $(\mathrm{F}=15.7, \mathrm{df}=1,45, \mathrm{p}<0.001)$ in the between subjects analysis (see figure 1 ). When analysed separately with $\mathrm{t}$ tests, the groups differed on all emotions, except happiness $(\mathrm{t}<1)$ - Anger $(\mathrm{t}=3.5, \mathrm{df}=45, \mathrm{p}=0.001)$, surprise $(\mathrm{t}=3.2, \mathrm{df}=45, \mathrm{p}=0.003)$, sadness $(\mathrm{t}=2.8, \mathrm{df}=45, \mathrm{p}=0.008)$, disgust $(\mathrm{t}=2.5, \mathrm{df}=45$, $\mathrm{p}=0.015)$, fear $(\mathrm{t}=2.4, \mathrm{df}=45, \mathrm{p}=0.02)$. The same effects were seen if the data were analysed with Mann-Whitney non parametric tests.

Notably, there was a significant correlation between ACE score and total emotion score (Pearson correlation $(\mathrm{pc})=0.839, \mathrm{n}=16, \mathrm{p}<0.001$ - see figure 1) and between the total DRS score and the total Ekman 60 score $(\mathrm{pc}=0.732, \mathrm{n}=23, \mathrm{p}<0.001)$. There was no correlation between total emotion score and disease duration $(\mathrm{pc}=0.14, \mathrm{n}=23, \mathrm{p}=\mathrm{ns})$ or stage of disease ( $\mathrm{pc}=0.083, \mathrm{n}=19, \mathrm{p}=\mathrm{ns})$.

\section{DISCUSSION}

Patients with PSP showed a clear deficit in recognizing facial emotions which was not attributable to a deficit in face perception or face recognition. However not all emotions were affected equally. Happiness recognition was not affected by PSP, indicating that the patients' poor performance cannot be attributed to a failure to understand the task. Preserved recognition of happiness might reflect preservation of a distinct neural substrate for this emotion, but we cannot exclude a ceiling effect or visuo-perceptual differences in the Ekman faces that facilitates recognition of this emotion. In contrast, the recognition of anger, disgust, surprise, fear and sadness were all affected. This wide ranging deficit, of predominantly negative emotions, is seen in other diseases, such as FTD [10, 17].

Previous research has shown that focal damage to the ventral frontal cortex results in impaired recognition of emotion [18]. This area is atrophic in PSP [19] and therefore it is possible that this atrophy underlies the emotion recognition deficits seen in this study. Alternatively, multiple brain regions necessary for normal recognition of multiple individual emotions may be affected. Previous studies have implicated the basal ganglia and the insula in the recognition of disgust [20] and the amygdala in the recognition of fear [21]. All of these regions are affected in PSP [22, 23].

The correlation between the degree of dementia (ACE and DRS scores) and patients' emotion recognition deficit, supports the hypothesis that generalised emotion and cognitive deficits form an integral syndrome in patients with PSP, secondary to neurodegeneration. 
Our patients divide into two groups - those with a score greater or lower than two standard deviations below the control mean for the emotion test (fig 1). Emotion recognition did not correlate with the duration of disease or with the stage of disease, implying that cognitive deficits are not inevitable as disease progresses. Rather, there are two subgroups of patients: one is associated with emotion/cognitive deficits, while the other subgroup is not. These subgroups (with or without emotion/cognitive deficits) refer to patients with a clinical PSP syndrome similar to Donker Kat [24]. This finding complements the distinction between PSP phenotype (Richardson syndrome) and a parkinsonian like phenotype (PSP-P) in pathologically proven cases of PSP seen by Williams [25]. Understanding this heterogeneity of PSP is an important step in the clinical care of patients and in progress towards effective therapies.

In summary, we report that patients with PSP have difficulty recognising a wide range of negative emotions. This is not a result of impaired face perception, but an integral part of the cognitive dysfunction associated with PSP. Emotion recognition problems correlate with cognitive function but not disease duration, implying a separate subtype of PSP. These findings offer an insight into a hitherto unrecognised deficit that is likely to contribute to difficulties in relationships and reduced quality of life for patients and carers.

\section{Acknowledgments}

\section{Funding:}

This work has been supported by the Medical Research Council UK (BCPG, AJC [U.1055.02.001.00001.01], JRH), The Wellcome Trust (JBR,[077029]), the Guarantors of Brain (BCPG), PSP Association (THB) and the Raymond and Beverley Sackler Foundation (BCPG).

Ethics approval:

Ethical approval was obtained from the Cambridge ethics committee based in Cambridge.

The Corresponding Author has the right to grant on behalf of all authors and does grant on behalf of all authors, an exclusive licence (or non exclusive for government employees) on a worldwide basis to the BMJ Publishing Group Ltd and its Licensees to permit this article (if accepted) to be published in the Journal of Neurology, Neurosurgery \& Psychiatry editions and any other BMJPGL products to exploit all subsidiary rights, as set out in our licence (http://jnnp.bmjjournals.com/ifora/licence.pdf).

\section{References}

1. Bak TH, et al. Cognitive bedside assessment in atypical parkinsonian syndromes. J Neurol Neurosurg Psychiatry. 2005; 76(3):420-2. [PubMed: 15716539]

2. Richardson JC, Steele J, Olszewski J. Supranuclear Ophthalmoplegia, Pseudobulbar Palsy, Nuchal Dystonia and Dementia. a Clinical Report on Eight Cases of "Heterogenous System Degeneration". Trans Am Neurol Assoc. 1963; 88:25-9. [PubMed: 14272249]

3. Litvan I, et al. Movement Disorders Society Scientific Issues Committee report: SIC Task Force appraisal of clinical diagnostic criteria for Parkinsonian disorders. Mov Disord. 2003; 18(5):467-86. [PubMed: 12722160]

4. Aarsland D, Litvan I, Larsen JP. Neuropsychiatric symptoms of patients with progressive supranuclear palsy and Parkinson's disease. J Neuropsychiatry Clin Neurosci. 2001; 13(1):42-9. [PubMed: 11207328]

5. Schrag A, et al. Health-related quality of life in patients with progressive supranuclear palsy. Mov Disord. 2003; 18(12):1464-9. [PubMed: 14673883]

6. Litvan I, et al. Natural history of progressive supranuclear palsy (Steele-Richardson-Olszewski syndrome) and clinical predictors of survival: a clinicopathological study. J Neurol Neurosurg Psychiatry. 1996; 60(6):615-20. [PubMed: 8648326] 
7. Sprengelmeyer R, et al. Loss of disgust. Perception of faces and emotions in Huntington's disease. Brain. 1996; 119(Pt 5)):1647-65. [PubMed: 8931587]

8. Breitenstein C, Daum I, Ackermann H. Emotional processing following cortical and subcortical brain damage: contribution of the fronto-striatal circuitry. Behav Neurol. 1998; 11(1):29-42. [PubMed: 11568400]

9. Dujardin K, et al. Deficits in decoding emotional facial expressions in Parkinson's disease. Neuropsychologia. 2004; 42(2):239-50. [PubMed: 14644109]

10. Keane J, et al. Face and emotion processing in frontal variant frontotemporal dementia. Neuropsychologia. 2002; 40(6):655-65. [PubMed: 11792405]

11. Litvan I, et al. Accuracy of clinical criteria for the diagnosis of progressive supranuclear palsy (Steele-Richardson-Olszewski syndrome). Neurology. 1996; 46(4):922-30. [PubMed: 8780065]

12. Hauw JJ, et al. Preliminary NINDS neuropathologic criteria for Steele-Richardson-Olszewski syndrome (progressive supranuclear palsy). Neurology. 1994; 44(11):2015-9. [PubMed: 7969952]

13. Mattis, S. Dementia Rating Scale. NFER Nelson; Windsor: 1988.

14. Mathuranath PS, et al. A brief cognitive test battery to differentiate Alzheimer's disease and frontotemporal dementia. Neurology. 2000; 55(11):1613-20. [PubMed: 11113213]

15. Calder AJ, et al. Facial Emotion Recognition after Bilateral Amygdala Damage: Differentially Severe Impairment of Fear. Cognitive Neuropsychology. 1996; 13(5):699-745.

16. Ekman, P.; Friesen, WV. Pictures of Facial Affect. Consulting Psychologists Press; Palo Alto, CA: 1976.

17. Lough S, et al. Social reasoning, emotion and empathy in frontotemporal dementia. Neuropsychologia. 2006; 44(6):950-8. [PubMed: 16198378]

18. Hornak J, et al. Changes in emotion after circumscribed surgical lesions of the orbitofrontal and cingulate cortices. Brain. 2003; 126(Pt 7):1691-712. [PubMed: 12805109]

19. Cordato NJ, et al. Clinical deficits correlate with regional cerebral atrophy in progressive supranuclear palsy. Brain. 2005; 128(Pt 6):1259-66. [PubMed: 15843423]

20. Sprengelmeyer R, et al. Neural structures associated with recognition of facial expressions of basic emotions. Proc Biol Sci. 1998; 265(1409):1927-31. [PubMed: 9821359]

21. Calder AJ, Lawrence AD, Young AW. Neuropsychology of fear and loathing. Nat Rev Neurosci. 2001; 2(5):352-63. [PubMed: 11331919]

22. Brenneis $\mathrm{C}$, et al. Voxel based morphometry reveals a distinct pattern of frontal atrophy in progressive supranuclear palsy. J Neurol Neurosurg Psychiatry. 2004; 75(2):246-9. [PubMed: 14742598]

23. Cordato NJ, et al. Regional brain atrophy in progressive supranuclear palsy and Lewy body disease. Ann Neurol. 2000; 47(6):718-28. [PubMed: 10852537]

24. Kaat L, et al. Frontal presentation in progressive supranuclear palsy. Neurology. 2007; 69(8):7239. [PubMed: 17709703]

25. Williams DR, et al. Characteristics of two distinct clinical phenotypes in pathologically proven progressive supranuclear palsy: Richardson's syndrome and PSP-parkinsonism. Brain. 2005; 128(Pt 6):1247-58. [PubMed: 15788542] 


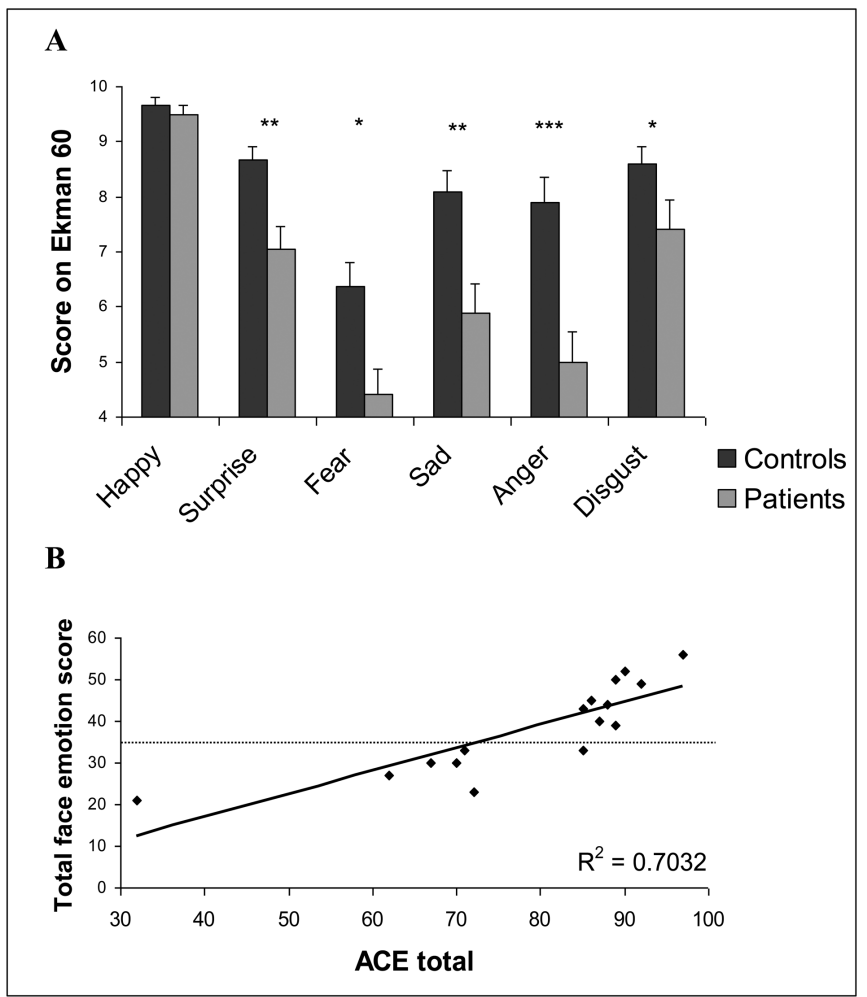

Figure 1.

A - Face emotion scores for the six basic emotions in the Ekman 60 test, for patients (grey) and healthy volunteers (black). Significant group differences are marked with asterixes (* $\mathrm{p}<0.05, * * \mathrm{p}<0.01, * * * \mathrm{p}<0.001)$. B - Correlation between ACE scores and total emotion score from the Ekman 60 test with the regression line (solid) showing a high degree of correlation $\left(\mathrm{R}^{2}=0.7032\right)$. The horizontal dashed line shows the value of the total emotion score two standard deviations below the mean for healthy volunteers. 


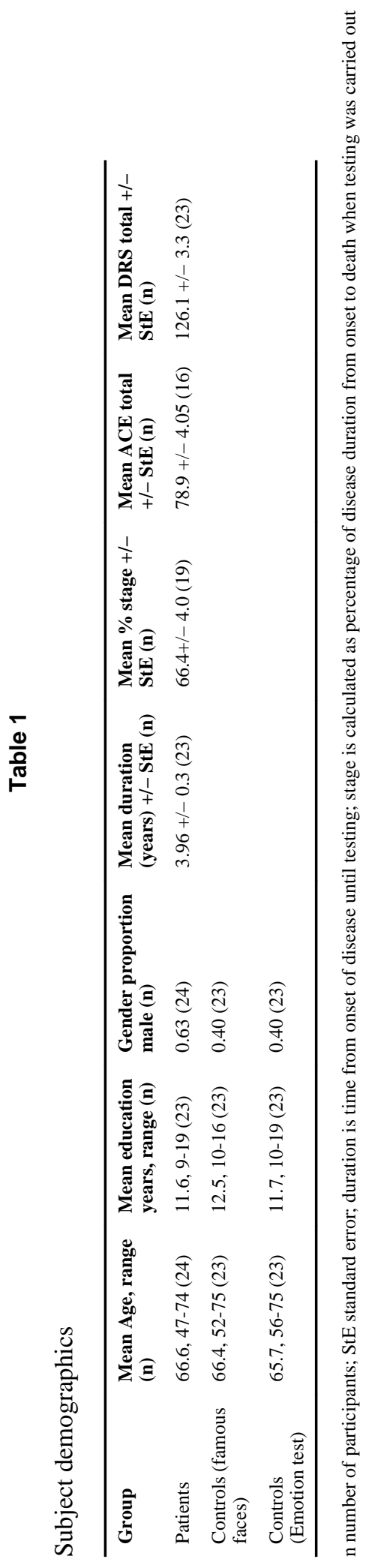

J Neurol Neurosurg Psychiatry. Author manuscript; available in PMC 2011 February 24. 